\title{
SIMULATION OF BEAM-ELECTRON CLOUD INTERACTIONS IN CIRCULAR ACCELERATORS USING PLASMA MODELS
}

\author{
A.Z. Ghalam, T. Katsouleas, USC, Los Angeles, USA \\ C. Huang, V. Decyk, W. B. Mori, UCLA, Los Angeles, USA
}

\begin{abstract}
In this paper we study the effect of low-density electron clouds on intense positively charged beams in circular accelerators. We apply one of the simulation tools we have been developing for the study of plasmabased accelerators to the problem of wake production and beam propagation in electron clouds. Particularly, we apply it to the electron cloud wakefields in the SPS proton synchrotron at CERN. We explore physics of beam-electron cloud interactions, particularly we present results on electron cloud effects on the long term beam dynamics. We present simulation results on the effects of bending magnets on the beam centroid oscillation, spot size and compare them to the case where bending magnets are not present.
\end{abstract}

\section{INTRODUCTION}

Electron clouds have been shown to be associated with limitations in particle accelerators performance in several of the world's largest circular proton and positron machines [1]. Electrons accumulate in the vacuum chamber where a positively charged bunched particle beam propagates because of a multipacting process which involves primary electron generation (e.g., from residual gas ionization) and their multiplication through secondary emission at the wall [2]. The detailed physics of electron cloud build up process has been extensively discussed elsewhere $[1,2,3]$. The presence of an electron cloud inside the beam chamber can deteriorate the vacuum [4], causing interference on the electrodes of beam pick up monitors and make the beam unstable via bunch-to-bunch or head-tail coupling $[5,6]$.

Although a number of beam-cloud interaction models that can satisfactorily explain many of the observations have been developed [7], the exact dynamics that leads to beam degradation (e.g., emittance growth, beam loss) is still under study. In particular, previous models have been limited in that they treat the cloud in an impulse approximation [8] (giving a single kick per turn) and they have not included conducting boundaries (and hence image forces of the cloud and beam charge) into the treatment of the beam dynamics.

In this paper we apply techniques from plasma wake field accelerator models to the study of the nonneutral plasma-beam interaction. We describe analytic and numerical models for the interaction between a positively charged beam and an electron cloud in a circular accelerator. Our model takes into account the effect of cloud image charges from the conducting beam pipe on the beam dynamics for the first time.
In second section we briefly describe the simulation model (QuickPIC). More detail can be found in [11]. In the third section we discuss different forces acting on the beam from the electron cloud and their effects on the beam dynamics. In the fourth section we study the effects of bending magnets on the cloud dynamics and its consequent impacts on the beam stability. Throughout the entire paper we adapt our physical parameters to the CERN-SPS parameters. These parameters can be found in Table 1 .

\section{BRIEF DESCRIPTION OF THE SIMULATION MODEL (QUICKPIC)}

QuickPIC is a 3-D PIC code using a quasi-static or frozen field approximation $[9,10]$. This approximation is specifically useful for studying wakes. It requires that the beam does not evolve significantly on the time scale that it takes the plasma to pass through it. The quasistatic approximation assumes that the wakes are functions of $z$-ct only and leads to equations for the wake potentials $\varphi$ and $\Psi=\varphi-A_{\|}$that involve only solving 2-D Poisson equations. The Poisson equations are solved on a 2-D slab of electron cloud with conducting boundary conditions. The wakes are stored and used to update the plasma in the slab and the slab is then pushed back a small step through the beam. After transiting the beam, the stored values of $\psi$ are used to find the force on the beam (treated as a 3-D PIC model) and it is pushed through a large step (of the order $\beta / 30$ ). As mentioned, the code is used for modeling plasma wakefield accelerators. In order to enable QuickPIC to simulate a bunch in a circular accelerator, some extra features needed to be added to correctly model the bunch evolution. In particular, betatron and synchrotron oscillations of the beam particles are introduced. These oscillations are due to the external fields of the magnets and RF power in the accelerator. Under the effect of these forces, individual particles (and the bunch as a whole, if off-centered) execute oscillations in all 3 spatial coordinates. All different aspects of the code have been fully discussed in [11].

\section{FORCES FROM THE ELECTRON CLOUD ACTING ON THE BEAM}

There are three different forces from the cloud acting on the beam. First, there is a direct force from the uniform cloud to the beam.

When a positively charged bunch passes through an electron cloud it sucks in the cloud towards its axis, creating cloud compression near the axis of the beam. The pinched cloud exerts a force to an unperturbed beam and focuses the beam toward its own axis. The 
situation becomes different when the beam is slightly tilted from its axis. In this case, the pinched cloud exerts a force on the tail of the beam so as to pull it back to the un-tilted axis.

When we have conducting boundary conditions, there is yet another restoring force acting on the displaced beam. The pinched cloud on the axis of the beam produces its own image charges of like sign of the beam in a conducting pipe and hence in a direction as to restore the beam to the pipe axis.

Expressions for these forces are given in [11].

Fig. 1 shows a beam off-centered $D \mathrm{~mm}$ from the pipe axis and slightly tilted $(\alpha)$ from the beam axis. Looking at the forces exerted on a particle at the tail of the beam, fist, there is a force from the uniform cloud density, this force contributes to the coherent betatron tune shift. Second, there is a force due to the image charges of the pinched cloud $\left(F_{c l_{-} y}\right)$, exerted on the tail of the beam pulling it toward the pipe axis. The last force exerted to the tail by electron cloud is a force due to direct pinched cloud $\left(\mathrm{F}_{\text {image_y }}\right)$ pulling it toward the beam axis. Based on this simple observation, we can say for any displacement of the beam $(D)$ there is always an equilibrium tile angle at which these two forces cancel each other at the tail of the beam. Therefore the long term propagation of the beam can be pictured as follows: No matter how the beam is initially perturbed, it will oscillate around the axis of the pipe with the tail slightly tilted toward the center line with respect to the head toward the axis.

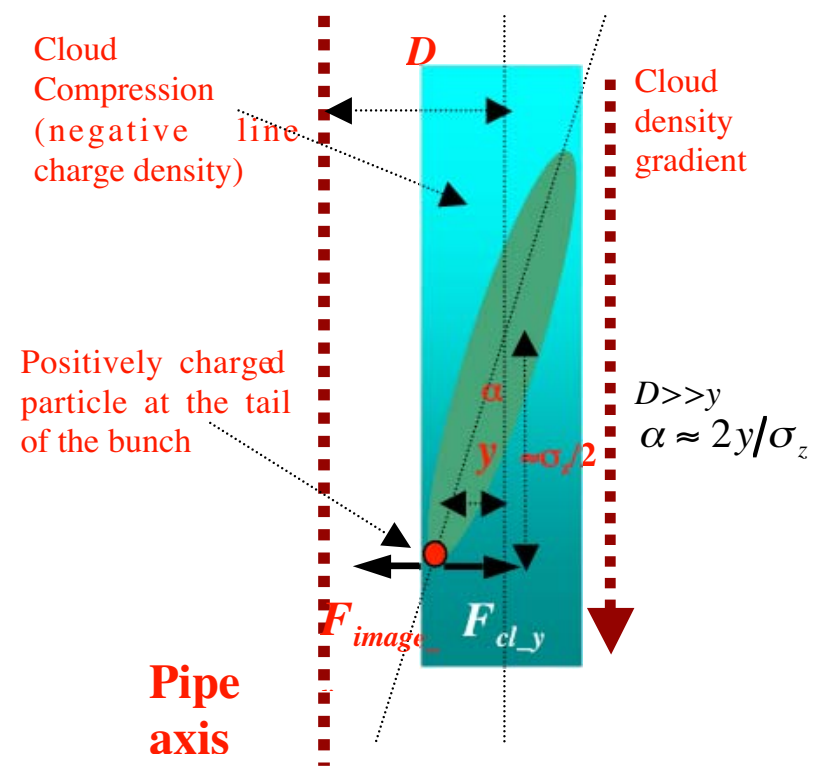

Fig.1. A cartoon showing a tilted beam and the electric field on the tail of the beam due to cloud compression on the axis

To verify the above hypothesis, two different simulations are performed by QuickPIC. In the first simulation, the beam is off-centered by $1 \mathrm{~mm}$ from the vertical axis as an initial perturbation to the beam while in the second run, the beam is tilted but not displaced in the vertical plane. Simulation results are shown in Fig.2.
The figure shows two snaps shot of the beam in each of these two runs. Fig.2-a and Fig.2-b show the initial beam, displaced and tilted from the pipe axis respectively and Fig.2-c and Fig.2-d are the corresponding snap shots of the beam after 18 and 35 turns of beam propagation over CERN-SPS ring. It is seen that the beam performs betatron oscillation around the pipe with its tail slightly tilted from its unperturbed axis.
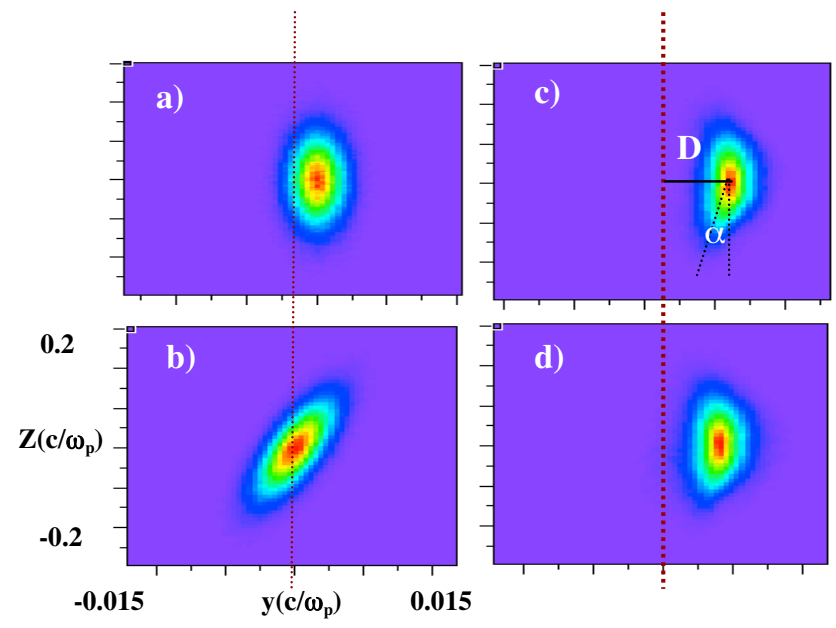

Fig.2. a) and b) initially displaced ( $1 \mathrm{~mm}$ ) and tilted beam respectively. c) A snap shot of the initially displaced beam after $0.4 \mathrm{~ms}$ (18 turns of SPS ring). d) A snap shot of the initially tilted beam after $0.6 \mathrm{~ms}(36$ turns of CERN-SPS ring)

A lower bound on the equilibrium tilt angle can be found by writing the total force by cloud on the tail and setting it to zero [11].

A detailed analysis of the beam dynamics and machine tune shift due to the electron cloud is discussed in [13].

\section{EFFECTS OF BENDING MAGNETS ON BEAM INSTABILITY}

In this section we study the effects of magnet systems on cloud dynamics and their consequent effects on the beam dynamics. We mentioned earlier that the cloud is sucked in toward the axis as the positive bunch passes. The situation is different when there is a magnetic field in the vertical direction. Electrons, rushing toward the axis feel a strong Lorentz force and perform cyclotron motion in horizontal plane with a small larmour radius. This cyclotron motion prevents them from being sucked in toward the axis and lowers the density of line charge on the pipe axis. Fig. 3 shows QuickPIC simulations for the cloud density in horizontal plane. Fig.3-a shows the density when there is a magnetic field of $0.117 \mathrm{~T}(\mathrm{CERN}-\mathrm{SPS}$ ring specification) in vertical direction and Fig.3-b is the cloud density when there is no magnetic field. As can be seen there is a high concentration of cloud density close to the axis in Fig.3-b while that of Fig.3-a is much shallower. 

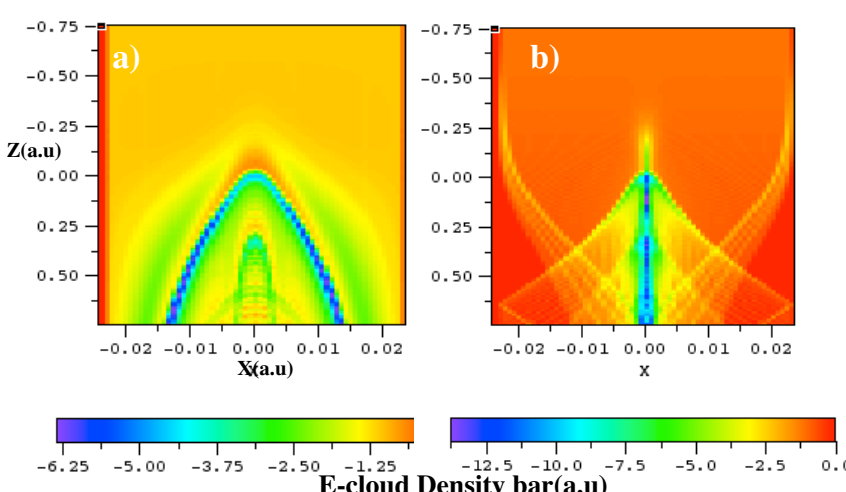

Figure 3: a) Cloud density in horizontal plane with $\mathrm{B}=0.117 \mathrm{~T}$. b) Cloud density in horizontal plane without magnetic field.

In order to study the impacts of the bending magnets on the beam dynamics, we look at the longterm propagation of the beam in CERN-SPS ring. Fig.4 shows the horizontal spot size of the beam for both cases of magnetic field on (Fig.4-a) and off (Fig.4-b). We perform the simulations over 125 turns of the ring. As can be seen there is a significant spot size growth in the no magnetic field case while there is almost no growth observed in the case with the bending magnets. We note that in the actual ring, the magnetic field is present in only $2 / 3$ of the ring.

The fact that the spot size growth is significantly suppressed, shows that bending magnets have a great stabilizing effect.

Further work is directed to modelling beam dynamics in this case of varying fields and cloud densities.
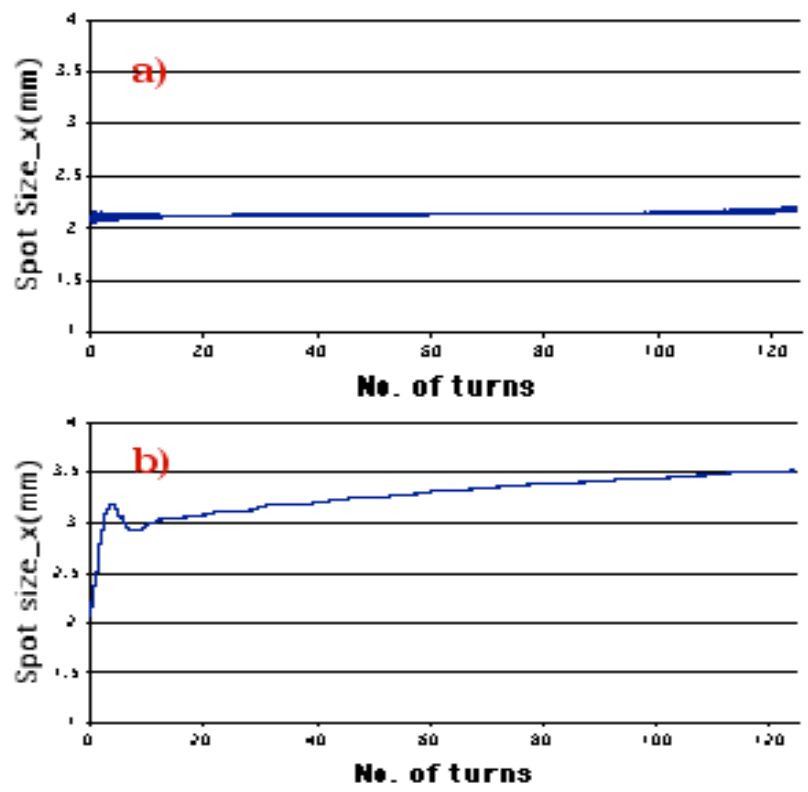

Figure 4: a) Horizontal spot size with $\mathrm{B}=0.117 \mathrm{~T}$. b) Horizontal spot size without magnetic field.

\section{ACKNOWLEDGEMENT}

This work is supported by:

USDOE \#DE-FG03-93ER40745, NSF-PHYS-

00787815, DE-FC02-01ER41192,DE-FG03-

92ER40727,DE-FC02-01ER41179,PHY-0078508
Table 1: Physical Parameters used in the Simulations

\begin{tabular}{|l|c|}
\hline Momentum Spread & $2.48 \mathrm{E}-3$ \\
\hline Beam Momentum $(\mathrm{Gev} / \mathrm{c})$ & 26 \\
\hline Circumference $(\mathrm{km})$ & 6.9 \\
\hline Horizontal Betatron Tune & 26.41 \\
\hline Vertical Betatron Tune & 26.41 \\
\hline Synchrotron Tune & 0.005 \\
\hline Electron Cloud Density $\left(\mathrm{cm}^{-3}\right)$ & $10^{6}-10^{7}$ \\
\hline Horizontal Spot Size $(\mathrm{mm})$ & 2 \\
\hline Vertical Spot Size $(\mathrm{mm})$ & 2 \\
\hline Bunch Length $(\mathrm{cm})$ & 30 \\
\hline Horizontal Box Size $(\mathrm{mm})$ & 80 \\
\hline Vertical Box Size $(\mathrm{mm})$ & 40 \\
\hline Bunch Population & $10^{11}$ \\
\hline Horizontal Emittance $(\mu \mathrm{m})$ & 0.1 \\
\hline Vertical Emittance $(\mu \mathrm{m})$ & 0.1 \\
\hline
\end{tabular}

\section{REFERENCES}

[1] H. Fukuma, K. Cornelis, F.-J. Decker, R. Macek, E. Metral, W. Fischer in Proceedings of ECLOUD'02, CERN, Geneva 15-18 April 2002, edited by G. Rumolo and F. Zimmermann, Yellow Report CERN-2002-001

[2] G. Rumolo, F. Ruggiero and F. Zimmermann, Phys. Rev. ST Accel. Beams, 4, 012801 (2001).

[3] M. T. F. Pivi and M. A. Furman, Phys. Rev. ST Accel. Beams 6, 034201(2003)

[4] A. Rossi, G. Rumolo, and F. Zimmermann, in Proceedings of ECLOUD'02, CERN, Geneva 15-18 April 2002, edited by G. Rumolo and F. Zimmermann, Yellow Report CERN-2002-001

[5] G. Arduini, K. Cornelis, W. Hoefle, G. Rumolo, and F. Zimmermann, in Proceedings

[6] K. Oide, "Observation and Cure of the Electron Cloud Effect at the KEKB Low Energy Ring", Chamonix XI, CERN SL-2001-003 (DI)

[7] K. Ohmi and F. Zimmermann, Phys. Rev. Lett. 85, 3821 (2000)

[8] G. Rumolo and F. Zimmermann, "Electron Cloud Simulations: Beam Instability and Wakefields" in Proceedings of ECLOUD'02, CERN, Geneva 15-18 April 2002, edited by G. Rumolo and F. Zimmermann, Yellow Report CERN-2002-001

[9] C. Huang et.al, proceedings, $18^{\text {th }}$ Annual Review of Progress in Computational Electromagnetics, p 557(2002)

[10] D.H. Whittum, Phys. Plasmas, 4, 1154(1997)

[11] G. Rumolo, A.Z. Ghalam et.al., " Electron Cloud Effects on Beam Dynamics evolution in a Circular Accelerator" Submitted to PR-STAB. 